\title{
RUIN PROBABILITIES UNDER AN OPTIMAL INVESTMENT AND PROPORTIONAL REINSURANCE POLICY IN A JUMP DIFFUSION RISK PROCESS
}

\author{
YIPING QIAN $^{1}$ and XIANG LIN ${ }^{\bowtie 2}$ \\ (Received 3 March, 2009; revised 5 November, 2009)
}

\begin{abstract}
In this paper, we consider an insurance company whose surplus (reserve) is modeled by a jump diffusion risk process. The insurance company can invest part of its surplus in $n$ risky assets and purchase proportional reinsurance for claims. Our main goal is to find an optimal investment and proportional reinsurance policy which minimizes the ruin probability. We apply stochastic control theory to solve this problem. We obtain the closed form expression for the minimal ruin probability, optimal investment and proportional reinsurance policy. We find that the minimal ruin probability satisfies the Lundberg equality. We also investigate the effects of the diffusion volatility parameter, the market price of risk and the correlation coefficient on the minimal ruin probability, optimal investment and proportional reinsurance policy through numerical calculations.

2000 Mathematics subject classification: primary 60G99; secondary 60G65, 91B30.

Keywords and phrases: ruin probability, proportional reinsurance, optimal investment policy, Lundberg's equality, Hamilton-Jacobi-Bellman equation.
\end{abstract}

\section{Introduction}

The ruin probability, when an insurance company has the possibility to invest part of its surplus into a financial market and to take proportional reinsurance for claims, has recently attracted increasing attention in the risk theory literature. Browne [1-3] first considers the approach where the surplus process is modelled by a Brownian motion with drift, and the risky asset follows a geometric Brownian motion. Without a budget constraint, the optimal investment strategy which minimizes the ruin probability is the investment of a constant amount of money in the risky asset, irrespective of the size of the surplus, and the corresponding minimal ruin probability is given by an exponential function. Liang [9], Schmidli [17], Promislow and Young [16],

\footnotetext{
${ }^{1}$ School of Business, Central South University, Yuelu Mountain, Changsha 410083, Hunan, PR China.

${ }^{2}$ School of Mathematics, Central South University, No. 22 South Shaoshan Road, Changsha 410075, Hunan, PR China; e-mail: xlin@csu.edu.cn.

(C) Australian Mathematical Society 2010, Serial-fee code 1446-1811/2010 \$16.00
} 
Taksar and Markussen [20] and Luo et al. [14] reconsider the model in Browne [1] with proportional reinsurance. They also derive a closed form expression for the optimal policy and the optimal value function. Hipp and Plum [7, 8] consider the classical risk model and assume that the surplus can be invested in a risky asset which follows a geometric Brownian motion without risk-free rate. They analyze the investment strategy to minimize the ruin probability, and then derive the HamiltonJacobi-Bellman equation corresponding to the problem and prove the existence of a solution and a verification theorem. Liu and Yang [13] and Yang and Zhang [22] reconsider the model in Hipp and Plum [8] with risk-free rate. Gaier et al. [5] consider the optimal investment problem for an insurance company under the framework of the classical risk process, where the claims have exponential moments. They obtain the Lundberg inequality for the minimal ruin probability. Lin [12] discusses ruin probability in a jump diffusion risk model where the surplus is invested in a risk-free asset and a risky asset. Schimidli [18] considers the model under which reinsurance and investment are allowed, the surplus is modeled by the classical risk process and a numerical procedure for solving the Hamilton-Jacobi-Bellman (HJB) equation is given.

Since it is very difficult to obtain an explicit expression for the ruin probability $\Psi(x)$ in the jump diffusion risk model, much of the literature focuses on the estimation of the ruin probability, especially upper bound estimation. For example, in Hald and Schmidli [6] and Liang and Guo [10, 11], the minimal ruin probability satisfies the Lundberg inequality

$$
\Psi(x) \leq C e^{-R x}
$$

where $C$ is a constant and $R$ is the adjustment coefficient.

In this paper we reconsider the ruin probabilities, optimal investment and proportional reinsurance policy of an insurance company whose surplus is modeled by a jump diffusion risk process as in Liang and Guo [11]. In [11] the insurance company can invest only in a risky asset whose price dynamics follows a geometric Brownian motion, and they obtain only an upper bound estimation of the minimal ruin probability. In this paper, the insurance company can invest in $n$ risky assets. We study a closed form expression for the minimal ruin probability, the optimal investment and the proportional reinsurance policy of the insurance company. We study the relationships between the minimal ruin probability and the investment, the reinsurance, the diffusion volatility parameter, the correlation coefficient and the market price of risk. We also investigate the effects of the diffusion volatility parameter, the correlation coefficient and the market price of risk on the optimal investment and proportional reinsurance policy through numerical calculations.

This paper is organized as follows. In Section 2, we give the model and some definitions and notation needed in this paper. In Section 3, we give the HJB equation and the verification theorem for the ruin probabilities and the optimal investment and proportional reinsurance policy. In Section 4, we study a closed form expression for 
the minimal ruin probabilities and the optimal investment and proportional reinsurance policy. In Section 5, we present some illustrative numerical results and economic analysis.

\section{The model}

To make a rigorous mathematical formulation of the problem, we assume that all processes and random variables are defined on a filtered probability space $(\Omega, \mathcal{F}, F, P)$ satisfying the usual conditions, that is, $F=\left\{\mathcal{F}_{t}, t \geq 0\right\}$ is right continuous and $P$-complete.

We consider the classical compound Poisson risk process perturbed by a diffusion or a jump diffusion process

$$
R(t)=x+c t-\sum_{k=1}^{N(t)} Y_{i}+\beta \tilde{W}(t)=x+c t-S(t)+\beta \tilde{W}(t),
$$

where $x \geq 0$ denotes the initial capital, $c>0$ is the rate of premium per unit time; $Y=\left\{Y_{k}, k=1,2, \ldots\right\}$ is a sequence of independent and identically distributed nonnegative random variables with a common distribution $F$ of finite mean $m_{1}$, where $Y_{k}$ denotes the amount of the $k$ th claim; $\{N(t), t \geq 0\}$ is a Poisson process with rate $\lambda>0$, representing the number of claims up to time $t ;\{\tilde{W}(t), t \geq 0\}$ is a standard Brownian motion; $\beta$ is a constant, representing the diffusion volatility parameter. $R(t)$ is the surplus of an insurance company at time $t$. In addition, $\left\{Y_{k}, k=1,2, \ldots\right\}$, $\{N(t), t \geq 0\}$ and $\{\tilde{W}(t), t \geq 0\}$ are mutually independent. As pointed out in Dufresne and Gerber [4], the perturbed compound Poisson risk process adds an uncertainty to the premium income or an additional uncertainty to the aggregate claims.

In proportional reinsurance, the reinsurer is required to pay a certain fraction of each claim, while in turn the insurer diverts the same or a larger fraction of all the premiums to the reinsurer. If the safety loading of the reinsurer and the insurer are the same, that is, if the fraction of the premium diverted to the reinsurer is the same as the fraction of each claim covered by the reinsurer, then the contract is called a cheap reinsurance; if the safety loading of the reinsurer is higher than that of the insurer, then the contract is called a noncheap reinsurance.

The proportional reinsurance level is associated with the value $1-a$, where $0 \leq$ $a \leq 1$ is called the risk exposure. If the risk exposure of the company is fixed, then the insurer pays proportion $a$ of each claim while the remaining proportion $1-a$ is paid by the reinsurer. To this end, the insurer diverts part of the premiums to the reinsurer at the rate $c_{1}(1-a)$ with $c_{1} \geq c$. As noted, when $c=c_{1}$, the reinsurance is cheap, while if $c_{1}>c$, it is noncheap. The corresponding surplus process of the insurance company becomes

$$
R(t, a)=x+\left[c-(1-a) c_{1}\right] t-a S(t)+\beta \tilde{W}(t) .
$$

In order for the net profit condition to be fulfilled, that is,

$$
c-(1-a) c_{1}-a \lambda m_{1}>0,
$$


we need

$$
a>\bar{a}=\frac{c_{1}-c}{c_{1}-\lambda m_{1}} .
$$

Otherwise, the probability of ruin will be one for any initial surplus $x \geq 0$.

In addition, there are $n$ risky assets available for the insurance company in the financial market. We use $\theta(t)=\left(\theta_{1}(t), \ldots, \theta_{n}(t)\right)^{\mathrm{T}}$ to denote the amounts of money of the surplus being invested in $n$ risky assets at time $t$. Here as subsequently T denotes transposition. The price processes are governed by the stochastic differential equation

$$
d S_{i}(t)=S_{i}(t)\left(\mu_{i} d t+\sum_{j=1}^{n} \sigma_{i j} d W_{j}(t)\right) \text { for } i=1, \ldots, n,
$$

where $\mu_{i} \geq 0, \sigma_{i j}>0$ are constants representing the expected instantaneous rate of return and the volatility of the risky asset $i$, respectively, and $W(t)=$ $\left(W_{1}(t), \ldots, W_{n}(t)\right)^{\mathrm{T}}$ is an $n$-dimensional standard Brownian motion.

We assume that $\left\{Y_{k}, k=1,2, \ldots\right\},\{N(t), t \geq 0\}$ and $\{W(t), t \geq 0\}$ are mutually independent, and the correlation coefficient of the Brownian motions $\tilde{W}, W_{j}$ is $\rho_{j}$, that is, $E\left[\tilde{W}(t) W_{j}(t)\right]=\rho_{j} t$, for $j=1,2, \ldots, n$. We also assume that $\sigma=\left(\sigma_{i j}\right)$ is a nonsingular matrix, and write $\Sigma=\sigma \sigma^{\mathrm{T}}, \rho=\left(\rho_{1}, \ldots, \rho_{n}\right)^{\mathrm{T}}$ and the market price of risk $\gamma=\sigma^{-1} \mu$, where $\mu=\left(\mu_{1}, \ldots, \mu_{n}\right)^{\mathrm{T}}$.

At any time $t \geq 0, a=a(t)$ and $\theta=\theta(t)$ are chosen by the insurance company. We denote $\pi(\cdot)=(a(\cdot), \theta(\cdot))$. Once the policy $\pi(\cdot)$ is chosen, the dynamics of the surplus process becomes

$$
d R(t, \pi)=\sum_{i=1}^{n} \theta_{i}(t) \frac{d S_{i}(t)}{S_{i}(t)}+d R(t, a)
$$

or, more explicitly,

$$
\begin{aligned}
d R(t, \pi)= & {\left[c-(1-a(t)) c_{1}\right] d t-a(t) d S(t)+\beta d \tilde{W}(t)+\sum_{i=1}^{n} \mu_{i} \theta_{i}(t) d t } \\
& +\sum_{i=1}^{n} \sum_{j=1}^{n} \theta_{i}(t) \sigma_{i j} d W_{j}(t), \\
R(0)= & x .
\end{aligned}
$$

The control policy $\pi(\cdot)$ is said to be admissible if $a(\cdot)$ and $\theta(\cdot)$ are predictable with respect to $F$ and, for each $t \geq 0$, the processes $a(\cdot)$ and $\theta(\cdot)$ satisfy the following conditions:

(1) $0 \leq a(t) \leq 1$;

(2) $P\left\{\int_{0}^{\infty} \theta(t)^{\mathrm{T}} \Sigma \theta(t) d t<\infty\right\}=1$.

The set of all admissible policies is denoted by $\Pi$.

The ruin time is defined as $\tau(\pi)=\inf \{t>0: R(t, \pi) \leq 0\}$; if $R(t, \pi)>0$ for all $t>0$, then $\tau(\pi)=\infty$. 
We define the infinite time ruin probability under policy $\pi$ when the initial surplus is $x$ by

$$
\Psi(x, \pi)=\mathbb{P}\{R(t, \pi) \leq 0 \text { for some } t \geq 0 \mid R(0)=x\}=\mathbb{P}\{\tau(\pi)<\infty \mid R(0)=x\} .
$$

The objective is to find the optimal value function (the minimal ruin probability)

$$
\Psi(x)=\inf _{\pi \in \Pi} \Psi(x, \pi),
$$

and the optimal policy $\pi^{*}$ such that $\Psi\left(x, \pi^{*}\right)=\Psi(x)$.

Denote

$$
h(r)=E\left[e^{r Y}\right]-1=\int_{0}^{\infty} e^{r y} d F(y)-1
$$

as the moment generating function of the claim size $Y$, and assume that there exists $r_{\infty}>0$ such that $h(r) \uparrow \infty$ when $r \uparrow r_{\infty}$ (we allow for the possibility $r_{\infty}=\infty$ ). It is easily seen that $h(0)=0$ and that $h$ is increasing, convex and continuous on $\left[0, r_{\infty}\right)$.

REMARK 1. If $n=1, a=q, c=(1+\theta) \lambda \mu$ and $c_{1}=(1+\eta) \lambda \mu$, this is the model of Liang and Guo [11].

\section{The Hamilton-Jacobi-Bellman equation and the verification theorem}

We can express the compound Poisson process $S(t)$ via

$$
d S(t)=\int_{0}^{\infty} y N(d t, d y) .
$$

The compensator of $N$, denoted by $\hat{N}(d t, d y)$, is given by

$$
\hat{N}(d t, d y)=E[N(d t, d y)]=\lambda F(d y) d t .
$$

$\tilde{N}(t, A)=N(t, A)-\hat{N}(t, A)$ is a martingale with respect to the filtration $F$ for $A \in \mathbf{B}_{0}$, where $\mathbf{B}_{0}$ is the family of Borel sets whose closure $A \subset R$ does not contain 0 .

We start with the associated HJB equation for the value function $\Psi$.

THEOREM 3.1. Assume that $\Psi(x)$ defined by (2.1) is twice continuously differentiable on $(0, \infty)$. Then $\Psi(x)$ satisfies the HJB equation

$$
\begin{gathered}
0=\inf _{\pi \in \Pi}\left\{\left[c-(1-a) c_{1}+\theta^{\mathrm{T}} \mu\right] \Psi^{\prime}(x)+\frac{1}{2}\left(\beta^{2}+2 \beta \theta^{\mathrm{T}} \sigma \rho+\theta^{\mathrm{T}} \Sigma \theta\right) \Psi^{\prime \prime}(x)\right. \\
\left.\quad+\lambda \int_{0}^{\infty}[\Psi(x-a y)-\Psi(x)] F(d y)\right\}, \quad x>0 .
\end{gathered}
$$

The proof of this theorem is standard (see Øksendal and Sulem [15, Chapter 3] or Schmidli [19]).

First, we give a very important lemma to prove the verification theorem, whose proof is identical to that of Lemma 6.1 in Taksar and Markussen [20]. 
LEMMA 3.2. Let $\eta(\pi, N)=\inf \{t>0: R(t, \pi) \geq N\}$, and

$$
\tau(\pi, N)=\min (\tau(\pi), \eta(\pi, N))=\inf \{t>0: R(t, \pi) \notin[0, N]\} .
$$

Then, for any $N>0$ and any policy $\pi$, we have that $\mathbb{P}\{\tau(\pi, N)<\infty\}=1$.

The following verification theorem is essential in solving the associated stochastic control problem. The proof is similar to that of Theorem 2.19 in Schmidli [19].

THEOREM 3.3. Suppose $\psi \in C^{2}$ is a decreasing convex function satisfying the HJB equation (3.1) subject to the boundary conditions

$$
\psi(0)=1, \quad \psi(\infty)=0 .
$$

Then the minimal ruin probability $\Psi(x)$ given by (2.1) coincides with $\psi$. Furthermore, if $\pi^{*}=\left(a^{*}, \theta^{*}\right)$ satisfies

$$
\begin{aligned}
0= & {\left[c-\left(1-a^{*}\right) c_{1}+\left(\theta^{*}\right)^{\mathrm{T}} \mu\right] \psi^{\prime}(x)+\frac{1}{2}\left(\beta^{2}+2 \beta\left(\theta^{*}\right)^{\mathrm{T}} \sigma \rho+\left(\theta^{*}\right)^{\mathrm{T}} \Sigma \theta^{*}\right) \psi^{\prime \prime}(x) } \\
& +\lambda \int_{0}^{\infty}\left[\psi\left(x-a^{*} y\right)-\psi(x)\right] F(d y) \quad \text { when } 0 \leq x<\infty,
\end{aligned}
$$

then the policy $\pi^{*}$ is an optimal policy, that is, $\psi(x)=\Psi(x)=\Psi\left(x, \pi^{*}\right)$.

\section{Ruin probability and optimal policy}

In this section we discuss the ruin probability and optimal policy for the insurance company.

By Theorem 3.1, we know that the minimal ruin probabilities and optimal policy satisfy the HJB equation (3.1).

It is obvious that $\Psi(0)=1$ due to the fluctuation property of the Brownian motion. From Equation (3.1) we know the optimal investment policy is given by

$$
\theta^{*}(t)=-\Sigma^{-1} \mu \frac{\Psi^{\prime}(x)}{\Psi^{\prime \prime}(x)}-\beta\left(\sigma^{-1}\right)^{\mathrm{T}} \rho .
$$

For $\Psi(0)=1$, we assume that the solution of Equation (3.1) is given by

$$
\Psi(x)=e^{-r x} \quad \text { for } x \geq 0 .
$$

The optimal investment policy is then given by

$$
\theta^{*}(t)=\frac{1}{r} \Sigma^{-1} \mu-\beta\left(\sigma^{-1}\right)^{\mathrm{T}} \rho .
$$

On substitution from (4.1) and (4.2) into (3.1), we have after simplification

$$
0=\inf _{a \in[\bar{a}, 1]}\{f(a)\}
$$


where

$$
\begin{aligned}
f(a)= & -c r+(1-a) r c_{1}+\frac{r}{2} \beta \rho^{\mathrm{T}} \sigma^{-1} \mu-\frac{1}{2} \mu^{\mathrm{T}}\left(\Sigma^{-1}\right)^{\mathrm{T}} \mu+\frac{r^{2}}{2} \beta^{2}\left(1-\rho^{\mathrm{T}} \rho\right) \\
& +\frac{r}{2} \beta \mu^{\mathrm{T}}\left(\sigma^{-1}\right)^{\mathrm{T}} \rho+\lambda \int_{0}^{\infty}\left(e^{r a y}-1\right) F(d y) .
\end{aligned}
$$

By setting $d f(a) / d a=0$, we have that

$$
c_{1}=\lambda \int_{0}^{\infty} y e^{r a y} F(d y)=\lambda h^{\prime}(a r) .
$$

Let $\eta=a r$. Then Equation (4.4) becomes

$$
h^{\prime}(\eta)=\frac{c_{1}}{\lambda} .
$$

By the properties of $h$, we know that Equation (4.5) has a unique positive solution $\delta$.

LEMMA 4.1. If $\delta$ is the unique positive solution of Equation (4.5), then $c_{1} \delta \geq \lambda h(\delta)$.

PROOF. Let $g(r)=c_{1} r-\lambda h(r)$. Then we have $g^{\prime}(r)=c_{1}-\lambda h^{\prime}(r)$, and

$$
g^{\prime \prime}(r)=-\lambda h^{\prime \prime}(r)=-\lambda \mathbb{E}\left[Y^{2} e^{r Y}\right] \leq 0 .
$$

By (4.5), we know that $g^{\prime}(\delta)=0$, so we have $g(\delta) \geq g(0)$, that is, $c_{1} \delta \geq \lambda h(\delta)$.

Now let $a$ be the root of the equation $d f(a) / d a=0$, so $\delta=a r$. Substituting $r=\delta / a$ into (4.3), we obtain

$$
\begin{aligned}
& \left(\frac{1}{2} \gamma^{\mathrm{T}} \gamma-\lambda h(\delta)+c_{1} \delta\right) a^{2}+\left(c \delta-c_{1} \delta-\frac{\beta}{2} \delta \rho^{\mathrm{T}} \gamma-\frac{\beta}{2} \delta \gamma^{\mathrm{T}} \rho\right) a \\
& \quad-\frac{\beta^{2}}{2}\left(1-\rho^{\mathrm{T}} \rho\right) \delta^{2}=0 .
\end{aligned}
$$

By Lemma 4.1, the positive solution of Equation (4.6) is given by

$$
\begin{aligned}
a(\delta)= & \frac{\left(c_{1}-c+\frac{1}{2} \beta \rho^{\mathrm{T}} \gamma+\frac{1}{2} \beta \gamma^{\mathrm{T}} \rho\right) \delta}{2\left(c_{1} \delta+\frac{1}{2} \gamma^{\mathrm{T}} \gamma-\lambda h(\delta)\right)} \\
& +\frac{\sqrt{\left(c_{1}-c+\frac{1}{2} \beta \rho^{\mathrm{T}} \gamma+\frac{1}{2} \beta \gamma^{\mathrm{T}} \rho\right)^{2} \delta^{2}+2 \beta^{2} \delta^{2}\left(1-\rho^{\mathrm{T}} \rho\right)\left[c_{1} \delta+\frac{1}{2} \gamma^{\mathrm{T}} \gamma-\lambda h(\delta)\right]}}{2\left(c_{1} \delta+\frac{1}{2} \gamma^{\mathrm{T}} \gamma-\lambda h(\delta)\right)}
\end{aligned}
$$

and the optimal proportional reinsurance policy is given by

$$
a^{*}=a(\delta) \wedge 1
$$


On substituting (4.7) into (4.3) and simplifying, we have that $r$ satisfies

$$
\frac{\beta^{2}}{2}\left(1-\rho^{\mathrm{T}} \rho\right) r^{2}+\left(\frac{1}{2} \beta \gamma^{\mathrm{T}} \rho+\frac{1}{2} \beta \rho^{\mathrm{T}} \gamma-c+c_{1}\right) r-c_{1} \delta-\frac{1}{2} \gamma^{\mathrm{T}} \gamma+\lambda h(\delta)=0
$$

for $a^{*}<1$ and

$$
\frac{\beta^{2}}{2}\left(1-\rho^{\mathrm{T}} \rho\right) r^{2}+\left(\frac{1}{2} \beta \gamma^{\mathrm{T}} \rho+\frac{1}{2} \beta \rho^{\mathrm{T}} \gamma-c\right) r-\frac{1}{2} \gamma^{\mathrm{T}} \gamma+\lambda h(r)=0 \quad \text { for } a^{*}=1
$$

From the above discussion, we obtain the following theorem.

THEOREM 4.2. Let $\delta$ be the positive solution of the equation $h^{\prime}(\delta)=c_{1} / \lambda$, and

$$
\begin{aligned}
a(\delta)= & \frac{\left(c_{1}-c+\frac{1}{2} \beta \rho^{\mathrm{T}} \gamma+\frac{1}{2} \beta \gamma^{\mathrm{T}} \rho\right) \delta}{2\left(c_{1} \delta+\frac{1}{2} \gamma^{\mathrm{T}} \gamma-\lambda h(\delta)\right)} \\
& +\frac{\sqrt{\left(c_{1}-c+\frac{1}{2} \beta \rho^{\mathrm{T}} \gamma+\frac{1}{2} \beta \gamma^{\mathrm{T}} \rho\right)^{2} \delta^{2}+2 \beta^{2} \delta^{2}\left(1-\rho^{\mathrm{T}} \rho\right)\left[c_{1} \delta+\frac{1}{2} \gamma^{\mathrm{T}} \gamma-\lambda h(\delta)\right]}}{2\left(c_{1} \delta+\frac{1}{2} \gamma^{\mathrm{T}} \gamma-\lambda h(\delta)\right)} .
\end{aligned}
$$

Then the optimal policy to minimize the ruin probability is

$$
\theta^{*}=\frac{1}{R} \Sigma^{-1} \mu-\beta\left(\sigma^{-1}\right)^{\mathrm{T}} \rho \quad \text { and } \quad a^{*}=a(\delta) \wedge 1,
$$

and the minimal ruin probability is given by

$$
\Psi(x)=e^{-R x},
$$

where $R$ satisfies the following conditions.

(i) If $a^{*}<1$, then $R$ is the unique positive solution of the equation

$$
\begin{aligned}
& \frac{\beta^{2}}{2}\left(1-\rho^{\mathrm{T}} \rho\right) r^{2}+\left(\frac{1}{2} \beta \gamma^{\mathrm{T}} \rho+\frac{1}{2} \beta \rho^{\mathrm{T}} \gamma-c+c_{1}\right) r-c_{1} \delta \\
& -\frac{1}{2} \gamma^{\mathrm{T}} \gamma+\lambda h(\delta)=0 .
\end{aligned}
$$

(ii) If $a^{*}=1$, then $R$ is the unique positive solution of the equation

$$
\frac{\beta^{2}}{2}\left(1-\rho^{\mathrm{T}} \rho\right) r^{2}+\left(\frac{1}{2} \beta \gamma^{\mathrm{T}} \rho+\frac{1}{2} \beta \rho^{\mathrm{T}} \gamma-c\right) r-\frac{1}{2} \gamma^{\mathrm{T}} \gamma+\lambda h(r)=0 .
$$

REMARK 2. In Liang and Guo [11], the weaker result is obtained where the minimal ruin probability is bounded above by $\Psi(x) \leq e^{-R_{J} x}$. 


\section{Numerical results and economic analysis}

In this section, we present some illustrative numerical results. For simplicity, we assume that there is only one risky asset, that is, $n=1$. We study the relationships between the minimal ruin probability and the investment, the reinsurance, the diffusion volatility parameter, the correlation coefficient and the market price of risk. We also investigate the effects of the diffusion volatility parameter, the correlation coefficient and the market price of risk on the optimal investment and proportional reinsurance policy through numerical calculations.

Suppose that the claim sizes are exponentially distributed with parameter $\alpha$, that is, the density function is $f(y)=\alpha e^{-y \alpha}$, where $y \geq 0$. Then,

$$
h(r)=\alpha \int_{0}^{\infty} e^{r y} e^{-y \alpha} d y-1=\frac{r}{\alpha-r} .
$$

Therefore, by $h^{\prime}(\delta)=c_{1} / \lambda$, we get the unique positive solution $\delta=\alpha-\sqrt{\alpha \lambda / c_{1}}$ and

$$
\begin{aligned}
a(\delta)= & \frac{\left(c_{1}-c+(\beta \mu \rho / \sigma)\right) \delta}{2\left(c_{1} \delta+\left(\mu^{2} / 2 \sigma^{2}\right)-(\lambda \delta /(\alpha-\delta))\right)} \\
& +\frac{\sqrt{\left(c_{1}-c+(\beta \mu \rho / \sigma)\right)^{2} \delta^{2}+2 \beta^{2} \delta^{2}\left(1-\rho^{2}\right)\left[c_{1} \delta+\left(\mu^{2} / 2 \sigma^{2}\right)-(\lambda \delta /(\alpha-\delta))\right]}}{2\left(c_{1} \delta+\left(\mu^{2} / 2 \sigma^{2}\right)-(\lambda \delta /(\alpha-\delta))\right)} .
\end{aligned}
$$

The optimal policy to minimize the ruin probability is

$$
\theta^{*}=\frac{\mu}{R \sigma^{2}}-\frac{\beta \rho}{\sigma}
$$

and

$$
a^{*}=a(\delta) \wedge 1
$$

and the minimal ruin probability is given by

$$
\Psi(x)=e^{-R x}
$$

where $R$ is the unique positive solution of the equation

$$
\frac{\beta^{2}}{2}\left(1-\rho^{2}\right) r^{2}+\left(\frac{\beta \mu \rho}{\sigma}-c+c_{1}\right) r-c_{1} \delta-\frac{\mu^{2}}{2 \sigma^{2}}+\frac{\lambda \delta}{\alpha-\delta}=0 \quad \text { for } a^{*}<1
$$

or

$$
\frac{\beta^{2}}{2}\left(1-\rho^{2}\right) r^{2}+\left(\frac{\beta \mu \rho}{\sigma}-c\right) r-\frac{\mu^{2}}{2 \sigma^{2}}+\frac{\lambda r}{\alpha-r}=0 \quad \text { for } a^{*}=1 .
$$

For the classical compound Poisson risk model that is perturbed by diffusion,

$$
R(t)=x+c t-\sum_{k=1}^{N(t)} Y_{i}+\beta \tilde{W}(t)
$$




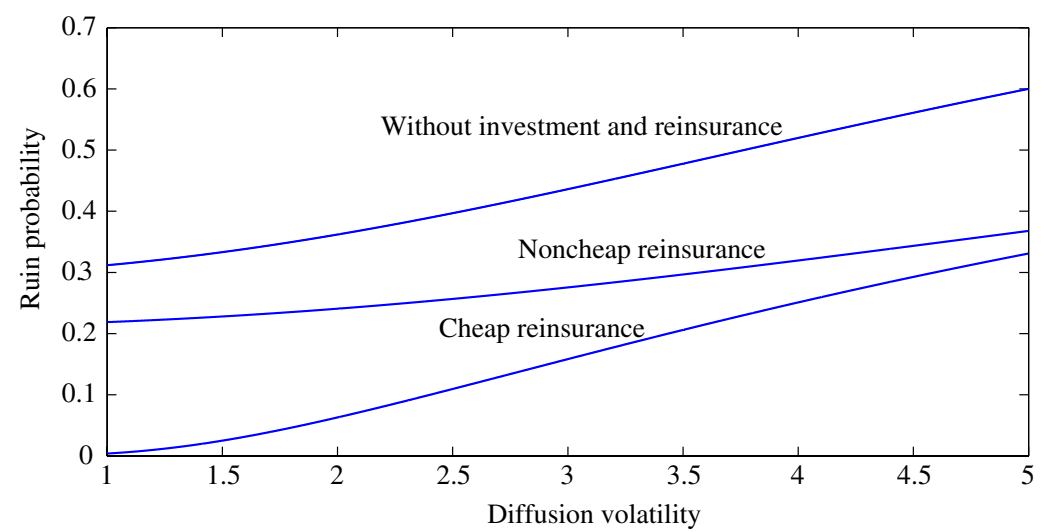

FIGURE 1. Effects of investment and reinsurance on the ruin probability.

we know by Dufresne and Gerber [4] or Wang and $\mathrm{Wu}$ [21] that the ruin probability satisfies

$$
\tilde{\Psi}(x)=C_{1} e^{-r_{1} x}+C_{2} e^{-r_{2} x},
$$

where

$$
\begin{gathered}
C_{1}=\frac{\beta^{2} r^{2}-2 r_{2} c}{\beta^{2}\left(r_{2}^{2}-r_{1}^{2}\right)-2 c\left(r_{2}-r_{1}\right)}, \quad C_{2}=-\frac{\beta^{2} r_{1}^{2}-2 r_{1} c}{\beta^{2}\left(r_{2}^{2}-r_{1}^{2}\right)-2 c\left(r_{2}-r_{1}\right)}, \\
r_{1}=\frac{1}{\beta^{2}}\left[\frac{1}{2} \alpha \beta^{2}+c-\sqrt{\left(\frac{1}{2} \alpha \beta^{2}-c\right)^{2}+2 \lambda \beta^{2}}\right] \quad \text { and } \\
r_{2}=\frac{1}{\beta^{2}}\left[\frac{1}{2} \alpha \beta^{2}+c+\sqrt{\left(\frac{1}{2} \alpha \beta^{2}-c\right)^{2}+2 \lambda \beta^{2}}\right] .
\end{gathered}
$$

Some examples are considered below. Examples 1-6 have the common feature that $\alpha=0.5, c=5$ and $\lambda=2$.

\subsection{Examples with $x=10$ and $\rho=0$}

EXAMPLE 1. We further assume that $\mu=0.10$ and $\sigma=0.20$ for $\beta \in[1,5]$ and calculate some ruin probabilities. We assume that $c_{1}=5$ and calculate the value of $\Psi_{1}(x)$ and $\tilde{\Psi}(x)$ by (5.3) and (5.4), respectively, and assume that $c_{1}=10$ to calculate $\Psi_{2}(x)$ by (5.3). The results are presented in Figure 1.

From Figure 1, we know that $\Psi_{1}(x)<\tilde{\Psi}(x)$ and $\Psi_{2}(x)<\tilde{\Psi}(x)$. That is, if the insurance company invests part of the surplus in risky assets and purchases proportional reinsurance for claims, no matter what the reinsurance policy (cheap or noncheap), it is possible to decrease the risk of ruin of the insurance company. We also observe that $\Psi_{1}(x)<\Psi_{2}(x)$, that is, the greater the reinsurance premium, the larger the minimal ruin probability, and as the cost of reinsurance increases it is natural to increase the risk of ruin. If we consider the minimal ruin probability as a function of $\beta$, 


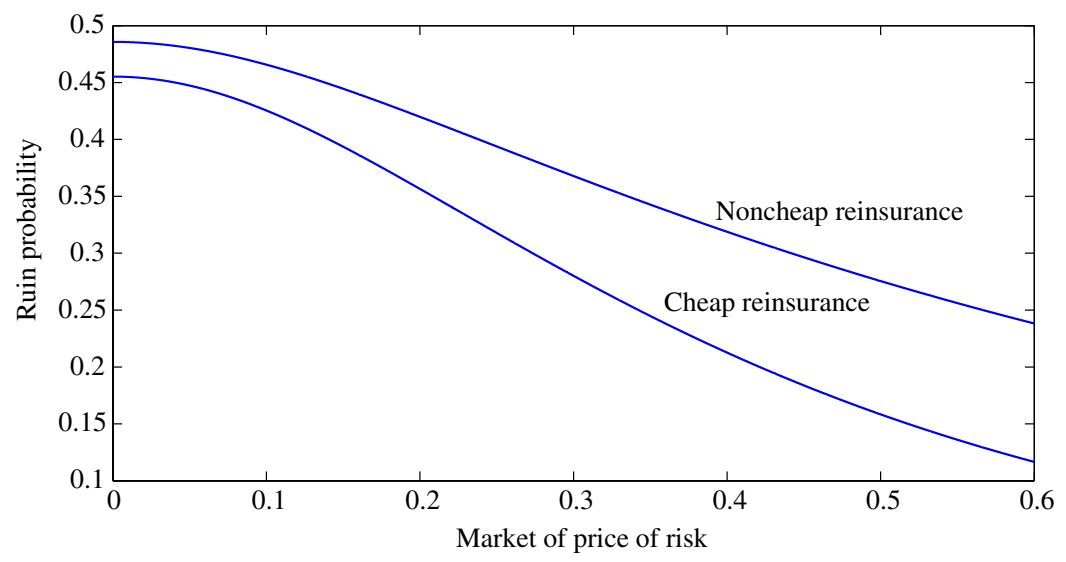

FIGURE 2. Effects of the market price of risk on the ruin probability.

then from Figure 1 we see that the minimal ruin probability is an increasing function of $\beta$, no matter what the reinsurance policy. As $\beta$ increases, the uncertainty risk of the insurance company will increase, so the risk of ruin will increase.

EXAMPLE 2. We further assume that $\beta=3$ for the market price of risk $\gamma=\mu / \sigma \in$ $[0,0.8]$. We calculate the value of the ruin probability $\Psi(x)$ by $(5.3)$ for $c_{1}=5$ and $c_{1}=10$ and present the results in Figure 2.

If we consider the minimal ruin probability as a function of the market price of risk $\gamma$, then from Figure 2 we see that the minimal ruin probability is a decreasing function of $\gamma$, no matter what the reinsurance policy. This simply states that as $\gamma$ increases, the risk of ruin of the insurance company will decrease. The reason for this is that as $\gamma$ increases, that is, the return of the unit risk is larger, the insurance company can obtain a greater yield from investment, so the risk of ruin will decrease.

\subsection{Examples with $\mu=0.10, \rho=-0.5,0,0.5$ and $\sigma=0.20$}

EXAMPLE 3. We assume that $x=10$ and $\beta \in[1.5,3]$, and calculate the value of the ruin probability $\Psi(x)$ by $(5.3)$ for $c_{1}=5$ and $c_{1}=10$. The results are presented in Figure 3 .

If we consider the minimal ruin probability as a function of the correlation coefficient $\rho$, then from Figure 3 we observe that the minimal ruin probability is an increasing function of $\rho$, no matter what the reinsurance policy. If $\rho$ is positive, then the risk of investment and the risk of the diffusion will aggregate; therefore, the total risk of the insurance company will increase, so the risk of ruin will increase. If $\rho$ is negative, then the risk of investment and the risk of diffusion will change in the opposite direction; therefore, the total risk of the insurance company will decrease, so the risk of ruin will decrease.

EXAMPLE 4 . We assume that $\beta \in[0.5,3]$ and calculate the optimal investment policy $\theta^{*}$ by $(5.1)$ for $c_{1}=5$ and $c_{1}=10$. The results are presented in Figure 4 . 


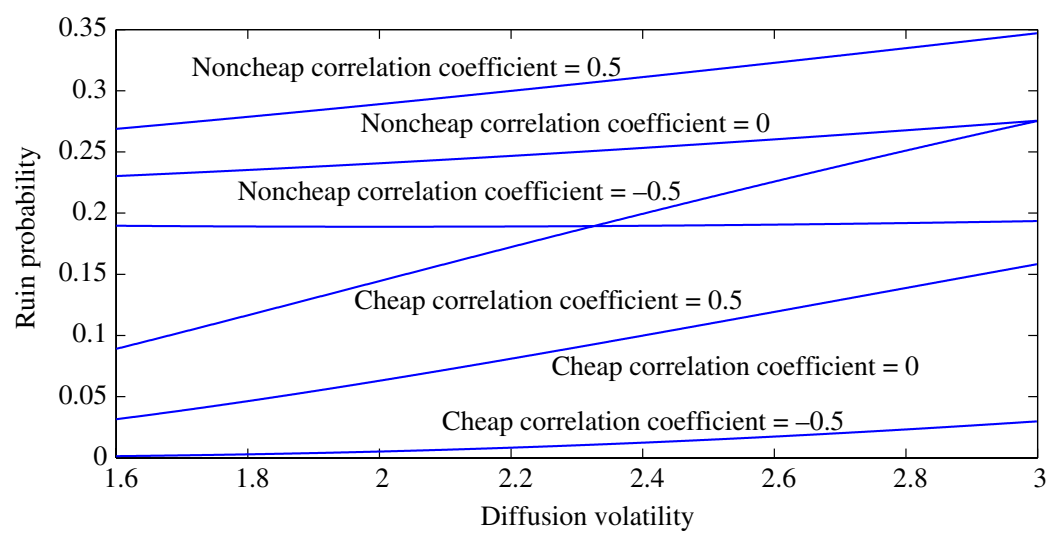

FIGURE 3. Effects of the diffusion volatility and the correlation coefficient on the ruin probability.

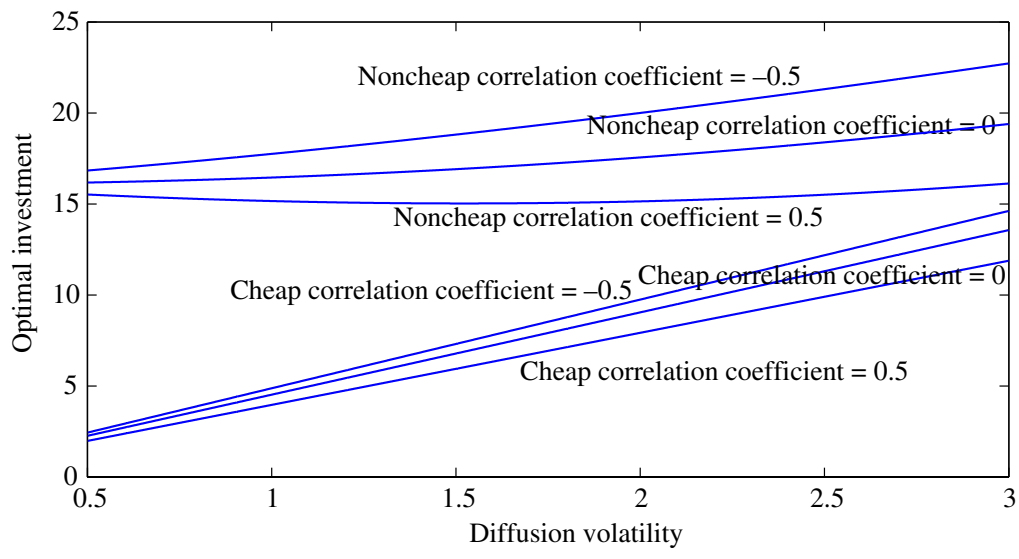

FIGURE 4. Effects of the diffusion volatility on the optimal investment.

If we consider the optimal investment policy as a function of the diffusion volatility parameter $\beta$, then from Figure 4 we observe that the optimal investment policy is an increasing function of $\beta$, no matter what the reinsurance policy. If we consider the optimal investment policy as a function of the correlation coefficient $\rho$, then from Figure 4 we see that the optimal investment policy is an decreasing function of the correlation coefficient $\rho$, no matter what the reinsurance policy. From Figure 4, we also know that the optimal investment policy is a increasing function of the reinsurance premium. This simply states that as the price of reinsurance increases, the insurer should invest more money in risky assets.

EXAMPLE 5. We assume that $\beta \in[1,5]$ and calculate the optimal proportional reinsurance policy $a^{*}$ by (5.2) for $c_{1}=5$ and $c_{1}=10$. The results are presented in Figure 5. 


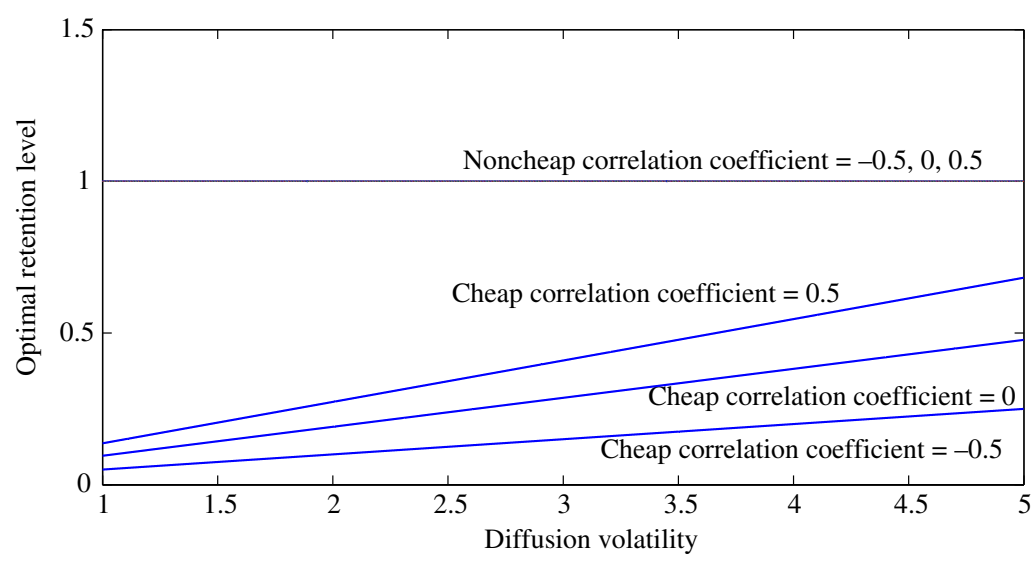

FIGURE 5. Effects of the diffusion volatility on the optimal retention level.

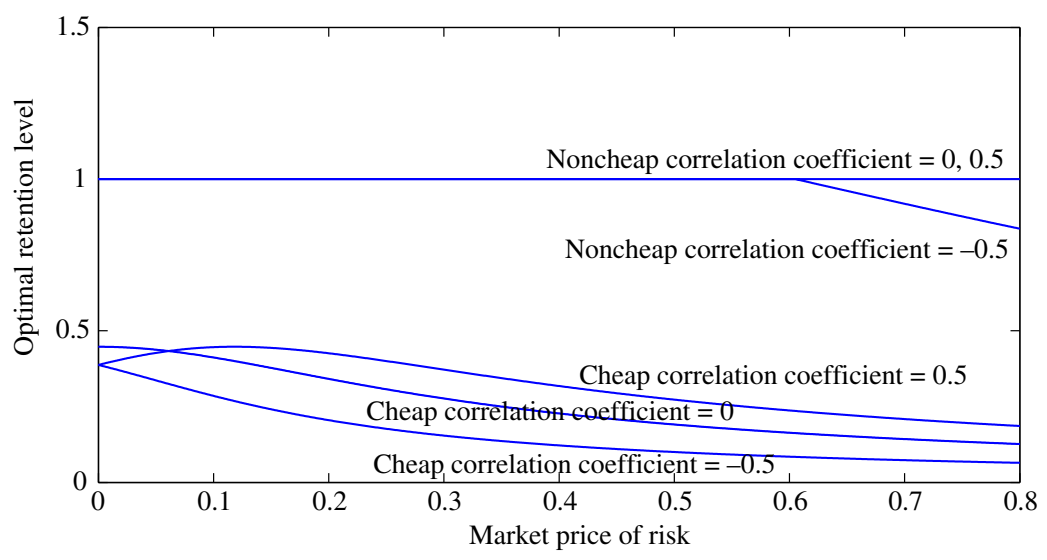

FIGURE 6. Effects of the market price of risk on the optimal retention level.

If we consider the optimal retention level as a function of the diffusion volatility parameter $\beta$, then from Figure 5 we observe that the optimal retention level is an increasing function of $\beta$ for any reinsurance policy. If we consider the optimal retention level as a function of the correlation coefficient $\rho$ then from Figure 5 we see that the optimal retention level is an increasing function of $\rho$ for any reinsurance policy. At the same time, from Figure 5 we also know that the optimal retention level is an increasing function of the reinsurance premium, and as the cost of reinsurance increases it is natural for the insurer to retain a greater share of each claim.

EXAMPLE 6. We assume that $\beta=2$ and $\gamma \in[0,0.8]$, and calculate the optimal proportional reinsurance policy $a^{*}$ by (5.2) for $c_{1}=5$ and $c_{1}=10$. The results are presented in Figure 6. 
From Figure 6, we see that as the market price of risk $\gamma$ is large, then the optimal retention level will decrease when $\gamma$ increases for any reinsurance policy.

\section{Acknowledgements}

This research was supported by the national Natural Science Foundation of China (project nos 10671212 and 10771216). We also thank the Technical Editor Jason Whyte for a careful reading of the paper and helpful suggestions that improved its presentation.

\section{References}

[1] S. Browne, "Optimal investment policies for a firm with a random risk process: exponential utility and minimizing the probability of ruin", Math. Methods Oper. Res. 20 (1995) 937-957.

[2] S. Browne, "Survival and growth with liability: optimal portfolio strategies in continuous time", Math. Methods Oper. Res. 22 (1997) 468-493.

[3] S. Browne, "Beating a moving target: Optimal portfolio strategies for outperforming a stochastic benchmark", Finance Stoch. 3 (1999) 275-294.

[4] F. Dufresne and H. U. Gerber, "Risk theory for the compound Poisson process that is perturbed by diffusion", Insurance Math. Econom. 10 (1991) 51-59.

[5] J. Gaier, P. Grandits and W. Schachermayer, "Asymptotic ruin probabilities and optimal investment", Ann. Appl. Probab. 13 (2003) 1054-1076.

[6] M. Hald and H. Schmidli, "On the maximization of the adjustment coefficient under proportional reinsurance", Astin Bull. 34 (2004) 75-83.

[7] C. Hipp and M. Plum, "Optimal investment for insurers", Insurance Math. Econom. 27 (2000) 215-228.

[8] C. Hipp and M. Plum, "Optimal investment for investors with state dependent income, and for insurers", Finance Stoch. 7 (2003) 299-321.

[9] Z. B. Liang, "Optimal proportional reinsurance for controlled risk process which is perturbed by diffusion", Acta Math. Appl. Sin. Engl. Ser. 23 (2007) 477-488.

[10] Z. B. Liang and J. Y. Guo, "Optimal proportional reinsurance and ruin probability", Stochastic Models 23 (2007) 333-350.

[11] Z. B. Liang and J. Y. Guo, "Upper bound for ruin probabilities under optimal investment and proportional reinsurance", Appl. Stoch. Models Bus. Ind. 24 (2008) 109-128.

[12] X. Lin, "Ruin theory for classical risk process that is perturbed by diffusion with risky investments", Appl. Stoch. Models Bus. Ind. 25 (2009) 33-44.

[13] C. S. Liu and H. Yang, "Optimal investment for an insurer to minimize its probability of ruin", N. Am. Actuar. J. 8 (2004) 11-31.

[14] S. Z. Luo, M. Taksar and A. Tsoi, "On reinsurance and investment for large insurance portfolios", Insurance Math. Econom. 42 (2008) 434-444.

[15] B. Øksendal and A. Sulem, Applied stochastic control in jump diffusions (Springer, Berlin, 2005).

[16] S. D. Promislow and V. R. Young, "Minimizing the probability of ruin when claims follow Brownian motion with drift", N. Am. Actuar. J. 9(3) (2005) 109-128.

[17] H. Schmidli, "Optimal proportional reinsurance policies in a dynamic setting", Scand. Actuar. J. 1 (2002) 55-68.

[18] H. Schmidli, "On minimizing the ruin probability by investment and reinsurance", Ann. Appl. Probab. 12 (2002) 890-907.

[19] H. Schmidli, Stochastic control in insurance (Springer, London, 2008).

[20] M. I. Taksar and C. Markussen, "Optimal dynamic reinsurance policies for large insurance portfolios", Finance Stoch. 7 (2003) 97-121. 
[21] G. Wang and R. Wu, "Some distributions for classical risk process that is perturbed by diffusion", Insurance Math. Econom. 26 (2000) 15-24.

[22] H. Yang and L. Zhang, "Optimal investment for insurer with jump-diffusion risk process", Insurance Math. Econom. 37 (2005) 615-634. 